\title{
The Intermittent Travelling Salesman Problem
}

\author{
Tu-San Pham, Pieter Leyman and Patrick De Causmaecker
}

November 12, 2018

\begin{abstract}
In this paper, we introduce a new variant of the Travelling Salesman Problem, namely the Intermittent Travelling Salesman Problem (ITSP), which is inspired by real world drilling/texturing applications. In this problem, each vertex can be visited more than once and there is a temperature constraint enforcing a time lapse between two consecutive visits. A branch-and-bound approach is proposed to solve small instances to optimality. We furthermore develop four Variable Neighbourhood Search metaheuristics which produce high quality solutions for large instances. An instance library is built and made publicly available.
\end{abstract}

\section{Introduction}

In this paper, we study a new variant of the Travelling Salesman Problem (TSP). The problem is inspired by industrial drilling or texturing applications where the temperature of the workpiece is taken into account. The processing causes the workpiece to locally heat up until it eventually melts the workpiece down. Therefore, the processing must be divided over time intervals, with time lapses for cooling down of the workpiece in between. This gives rise to an optimisation problem of finding the best schedule to process the whole workpiece in the shortest time while respecting temperature constraints. In this paper, we study a simplified version of the problem where we assume linearity with identical and constant cooling and heating speeds. We model the problem as a variant of the Travelling Salesman Problem in which some vertices are visited multiple times and there is a time lapse between two consecutive visits of a vertex, which is caused by the maximum temperature constraint, hence the name Intermittent Travelling Salesman Problem (ITSP).

The TSP is a well-studied problem in the field of combinatorial optimization with several variants. Extended reviews of the TSP can be found in e.g. Laporte (1992) and Johnson and McGeoch (1997). To the best of our knowledge, the ITSP is the first variant with intermittent constraints. The ITSP bears similarities to the Vehicle Routing Problem (VRP) family. The following problems share similarities with the ITSP. We point out the important differences.

- The TSP with multiple visits (TSPM) (Gutin and Punnen, 2006): In the TSPM, a vertex must be visited a number of times. However, unlike the ITSP, each visit is not associated with a processing time and there is no time constraint between visits. 
- The TSP with Time Windows (TSPTW) (Dumas et al., 1995): The TSPTW has time constraints associated with each vertex in the form of a time window. In contrast to the ITSP, the TSPTW does not consider multiple visits and the time constraints apply only for individual vertices.

- The Inventory Routing Problem (IRP) (Campbell et al., 1998): Each vertex (retailer) has a periodical (e.g. daily) requirement and an inventory capacity that should not be exceeded. The problem consists of finding the best shipping policy to deliver a product from a common supplier to several retailers, subject to the vehicle capacity constraints, product requirements and inventory capacities of the retailers. The inventory constraints are similar to the temperature constraints, be it that in the IRP, vehicle travelling routes are within one discrete period (e.g. a day). Therefore, route scheduling does not affect retailer inventory levels, in contrast with the ITSP where travelling time directly affects vertex temperature.

In this paper, we provide a detailed description of the ITSP and analyse its properties. We study a decomposition model, a branch-and-bound approach and a metaheuristic. A decomposition model is proposed including a Mixed Integer Programming (MIP) and a Linear Programming (LP) formulation for the subproblems. These are integrated in a branch-and-bound approach shown to solve small instances to optimality. We then develop a metaheuristic approach for the larger instances. Two constructive heuristics are proposed. Those two heuristics and two perturbation strategies are integrated in a Variable Neighborhood Search (VNS) framework resulting in four VNS metaheuristics. We analyse problem difficulty and create a problem library as a foundation for further research.

The structure of the paper is as follows: section 2 describes the ITSP in detail and analyses its difficulty. Section 3 introduces the building blocks of the branchand-bound approach, namely a lemma, two subproblems and two corresponding mathematical models. Section 4 is devoted to the Variable Neighborhood Search approach. Experimental results are given in section 5 and finally a conclusion and future work follow in section 6 .

\section{Problem description}

We consider the problem of visiting a number of vertices $v \in V$ with a given distance matrix $c_{v u} \geq 0$ defined for every pair of vertices $(v, u) \in V \times V$ with $c_{v v}=0$. We assume the triangle inequality holds. Each vertex $v \in V$ needs to be processed for a certain time $d_{v}$. Let $t, t_{s}$ and $t_{e}$ be the current time, the beginning and the end of the current contiguous period of processing respectively. While being processed, the temperature of a vertex rises linearly, $T_{v}(t)=T_{v}\left(t_{s}\right)+\left(t-t_{s}\right)$. While not being processed, the temperature cools linearly, $T_{v}(t)=\max \left(T_{v}\left(t_{e}\right)-t, 0\right)$ with a minimum of 0 , below which no cooling occurs. The maximum temperature of a vertex is $B$, beyond which no processing is allowed. We assume that at the beginning of the processing, the temperature of each vertex is 0 . The ITSP asks for the processing schedule of shortest total completion time.

We introduce some terminologies used throughout the paper: 
- A processing-step is a processing interval without interruption at a vertex.

- A visit consists of one or more processing-steps at one vertex without any visits to other vertices in between.

The term 'seconds' is used to indicate time units. Due to the maximum temperature constraint, a processing-step can never exceed $B$ seconds. For example, a vertex $v$ needs to be processed for 20 seconds while the maximum temperature $B$ allowed is 10 degrees. We let the increase in temperature during processing be one degree per second. Given this linear increase and decrease, $v$ must be processed in at least two blocks of 10 seconds with a time lapse of at least 10 seconds in between. Hence, we can say $v$ is processed using at least two processing-steps. If between those two processing-steps there is only waiting time and no processing-steps at other vertices, we say node $v$ is processed by one visit. Otherwise, we say $v$ is processed by two visits.

For example, if the sequence of processing for an instance consisting of 2 vertices is $A-A-B$ with a waiting time between two processing intervals of $A$, we say $A$ is processed in one visit and this visit includes two processing-steps.

We hereby discuss the number of processing-steps in optimal solutions. The duration of a processing-step must be less than or equal to $B$ time units where $B$ is the maximum temperature allowed. This results in the minimum number of processing-steps required for a vertex $v$ (denoted by $\phi_{v}$ ), with processing time $d_{v}$, given by:

$$
\phi_{v}=\left\lceil d_{v} / B\right\rceil
$$

In some instances where the maximum temperature is significantly smaller than the average travelling time between vertices, we may have to divide the processing of $v$ into more than $\phi_{v}$ processing-steps.

Such an example can be seen in figure 1. In this instance, vertex $A$ has a processing time $d_{A}=38$ seconds while four other vertices all have a processing time of 1 second. The maximum temperature $B$ is 10 degrees. There are two solutions considered: $S_{1}$ where $A$ is processed in 5 processing-steps (denoted by $A_{i}, i=\{1, . ., 5\}$, with the corresponding processing times denoted by $p_{A_{i}}$ ); and $S_{2}$ where $A$ is processed in 4 processing-steps $\left(A_{i}, i=\{1, . ., 4\}\right)$, i.e. the minimum number of processing-steps required, with positive waiting times at the third and the fourth processing-steps $\left(w_{A_{3}}, w_{A_{4}}>0\right)$. In the figure, $[v]$ denotes the tour time to move from vertex $A$ to vertex $v$, process $v$ and then come back to $A$. Despite the larger number of processing-steps, $S_{1}$ has a better objective value (66) than $S_{2}$ (69) due to no waiting time between processingsteps.

\section{Branch-and-bound approach}

\subsection{Mathematical models and lemmas}

In this section, the building blocks of the branch-and-bound approach, namely a lemma, two subproblems and two mathematical models to solve the subproblems, are introduced. 

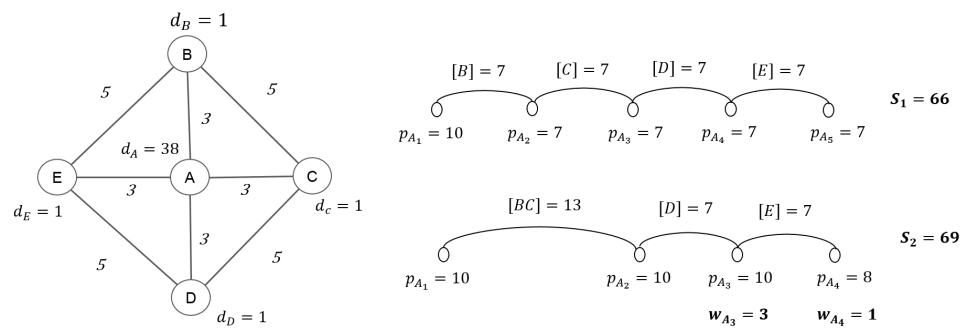

Figure 1: Sample instance illustrating a larger number of processing-steps may yield a better solution.

\subsubsection{Subproblem 1: fixed number of processing-steps}

Given an instance and a fixed number $m$ of processing-steps, the optimal solution can be obtained using the MIP model below.

Consider a set of processing-steps $Q=\{0, . ., m-1\}$, each processing-step $j \in Q$ is associated with a vertex $v \in V . d_{v}$ is the required processing time of vertex $v$ and $c_{v u}$ is the cost (in time units) to travel from vertex $v$ to vertex $u$.

The model consists of the following variables:

- $y_{j v}$ (binary): $y_{j v}=1$ iff vertex $v$ is processed during processing-step $j$.

- $x_{j v u}$ (binary): $x_{j v u}=1$ iff vertex $v$ is processed during processing-step $j$ and vertex $u$ is processed during processing-step $j+1$.

- $s_{j v}$ (continuous): the processing time of processing-step $j \in Q$ at vertex $v \in V$.

- $t_{j}$ (continuous): the time at the beginning of processing-step $j \in Q$.

- $q_{j v}$ (continuous): the temperature of vertex $v$ at the beginning of processingstep $j \in Q$.

The objective is to minimize the total completion time, which is the sum of the starting time of the final processing-step and its processing time. The model is as follows:

$$
\text { Minimize } t_{m-1}+\sum_{v \in V} s_{m-1, v}
$$

subject to

$$
\begin{array}{cr}
y_{j v}=\sum_{u \in V} x_{j v u} & \forall j \in Q, v \in V \\
y_{j v}=\sum_{u \in V} x_{j-1, u v} & \forall j \in Q \mid j>0, v \in V \\
\sum_{v \in V} y_{j v} \leq 1 & \forall j \in Q \\
y_{0,0}=y_{m-1,0}=1 &
\end{array}
$$




$$
\begin{array}{rr}
t_{j+1} \geq t_{j}+\sum_{v \in V} s_{j v}+\sum_{v, u \in V} c_{v u} x_{j v u} & \forall j \in Q \mid j \neq m-1 \\
\sum_{j \in Q} s_{j v}=d_{v} & \forall v \in V \\
q_{j+1, v} \geq q_{j v}+2 s_{j v}-\left(t_{j+1}-t_{j}\right) & \forall j \in Q \mid j \neq m, \forall v \in V \\
q_{j v}+s_{j v} \leq B & \forall j \in Q, v \in V \\
s_{j v} \leq B y_{j v} & \forall j \in Q, v \in V \\
x_{j v u} \in\{0,1\} & \forall j \in Q, v, u \in S \\
y_{j v} \in\{0,1\} & \forall j \in Q, v \in V \\
0 \leq s_{j v} \leq B & \forall j \in Q, v \in V \\
0 \leq t_{j} & \forall j \in Q \\
0 \leq q_{j v} \leq B & \forall j \in Q, v \in V
\end{array}
$$

where (2) and (3) are linking constraints between $x$ and $y$. (4) are flow constraints while (5) imposes the starting and ending depot (vertex 0). Constraints (6) define arrival times. Constraints (7) ensure total processing times of vertices. Constraints (8) define arrival temperature of processing-steps. This equation comes from the linear increase and decrease of temperature, which results in the temperature at the beginning of the processing-step $j+1$ being defined as $q_{j+1, v}=\max \left(q_{j v}+s_{j v}-\left(t_{j+1}-\left(t_{j}+s_{j v}\right)\right), 0\right)$. (9) are temperature constraints and (10) are linking constraints between $s$ and $y$. The rest are range constraints.

\subsubsection{Subproblem 2: fixed sequence of visits}

Given a fixed sequence of visits, the optimal completion time can be obtained in polynomial time using a Linear Programming (LP) model. Note that, unlike the MIP model in subproblem 1, this model only needs to consider visits eventually aggregating a number of processing-steps.

An example of a fixed sequence of visits can be found in figure 2. The sequence has 4 visits, each of which is associated with a vertex, a processing time and a waiting time. Visit 0,1 and 3 include a single processing-step whose processing time is smaller than or equal to the maximum temperature $B$ (10 time units) while visit 2 includes 2 processing-steps with total processing time $p_{2}=13>B$. Since its total processing time is larger than the maximum temperature, a waiting time of $w_{2}=B-p_{2}=3$ time units is required.

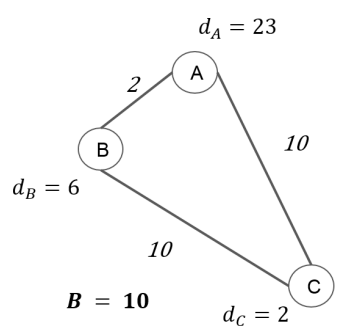

\begin{tabular}{|c|c|c|c|c|}
\hline Visit $i$ & 0 & 1 & 2 & 3 \\
\hline Vertex $v$ & $\mathrm{~A}$ & $\mathrm{~B}$ & $\mathrm{~A}$ & $\mathrm{C}$ \\
\hline$p_{i}$ & 10 & 6 & 13 & 2 \\
\hline$w_{i}$ & 0 & 0 & 3 & 0 \\
\hline
\end{tabular}

Figure 2: A fixed sequence of visits. 
Given is a fixed sequence $\Sigma$ of $n_{\Sigma}$ visits in which every vertex $v \in V$ is visited at least once. Let $w_{i}, p_{i}$ and $q_{i}$ represent the waiting time, the total processing time and the temperature at arrival of visit $i\left(i \in\left\{0, . ., n_{\Sigma}-1\right\}\right)$. Given a visit $i$ associated with vertex $v \in V$, pre $_{i}$ denotes the previous visit associated with the same vertex (in the example in figure 2, pre $_{2}=0$ ). $\Pi_{v}$ is the set of visits associated with vertex $v \in V$. An optimal visiting schedule of $\Sigma$, i.e. processing times and waiting times of its visits, can be calculated using the LP model as follows.

$$
\operatorname{Minimize} \sum_{i=0}^{n_{\Sigma}-1} w_{i}
$$

subject to

$$
w_{i} \geq q_{i}+p_{i}-B \quad i \in\left\{0, \ldots, n_{\Sigma}-1\right\}
$$

$$
\sum_{i \in \Pi_{v}} p_{i}=d_{v}
$$

$q_{i} \geq q_{\text {pre }_{i}}+\left(p_{\text {pre }_{i}}-w_{\text {pre }_{i}}\right)-c_{\text {pre }_{i}, \text { pre }_{i}+1}-\sum_{k=p r e_{i}+1}^{i-1}\left(c_{v_{k} v_{k+1}}+p_{k}+w_{k}\right) \quad i \in\left\{0, . ., n_{\Sigma}-1\right\}$

$$
w_{i}, p_{i}, q_{i} \geq 0 \quad i \in\left\{0, \ldots, n_{\Sigma}-1\right\}
$$

The goal function (16) minimizes the total waiting time. Constraints (17) come from the statement of vertex temperature, namely that temperature upon arrival $\left(q_{i}\right)+$ processing time $\left(p_{i}\right)$ - waiting time $\left(w_{i}\right.$, corresponds with cooling down of vertex) should never be larger than the maximum temperature $(B)$. Constraints (18) make sure each vertex is processed exactly its required amount of time. Constraints (19) limit the temperature at the start of the $i^{\text {th }}$ visit from below by considering the arrival temperature $\left(q_{p_{r} e_{i}}\right)$, total processing time and waiting time $\left(p_{\text {pre }_{i}}-w_{\text {pre }_{i}}\right)$ of its previous visit and the tour time (travelling times plus processing times and waiting times) of all visits between pre $_{i}$ and $i$ (the remaining part of the equation). The program can be solved in polynomial time in the number of variables which is proportional to $n_{\Sigma}$. 
Adding a visit to a sequence results in an increase in its total travelling time. For any three vertices $v, x, y \in V$, let $t_{v x y}$ represent the travelling time increased when a visit of $v$ is added between two consecutive visits of $x$ and $y$ in a sequence:

$$
t_{v x y}=c_{x v}+c_{v y}-c_{x y}
$$

We have the following:

Lemma 1. If, for a given sequence $\Sigma$, the optimal total waiting time $W_{\Sigma}=$ $\sum_{i=0}^{n_{\Sigma}-1} w_{i}$ is less than or equal to $\min _{v, x, y \in V}\left\{t_{v x y}\right\}$, no extension of $\Sigma$ generated by adding an extra visit can produce a shorter completion time.

Proof. Adding an extra visit increases the total travelling time by at least $\min _{v, x, y \in V}\left\{t_{v x y}\right\}$ and reduces the waiting time by at most $W_{\Sigma}$. Given the condition $W_{\Sigma} \leq \min _{v, x, y \in V}\left\{t_{v x y}\right\}$, the total processing time is increased.

\subsection{Branch-and-bound approach}

Based on the above subproblems and lemma, a branch-and-bound approach is proposed to solve the ITSP. We first present the data structure used in the algorithm.

Each node of the search tree is a sequence of visits. Given a sequence, its schedule, i.e. processing time and waiting time at each visit, is obtained using the LP model from subproblem 2. Since the total processing time is the same for all nodes in the search tree, we omit it to simplify the model. A node $\psi$ in the search tree is a direct child of a node $\chi$ if $\psi$ is created by inserting one more visit into the sequence of $\chi$. Since the triangle inequality holds, given a node $\chi$ with the total travelling time $T_{\chi}$, the total travelling time of each of its descendants is larger than or equal to $T_{\chi}$. Hence, we can say $T_{\chi}$ is a valid lower bound for $\chi$ and all its descendants.

$$
L B_{\chi}=T_{\chi}
$$

A node $\chi$ can be safely pruned when $L B_{\chi} \geq U B$ where $U B$ is the global upper bound of the search tree, i.e. $T_{\chi^{*}}+W_{\chi^{*}}$ where $\chi^{*}$ is the best solution so far. A node is also pruned when the condition in lemma 1 is satisfied.

At the beginning of the search, the MIP model in subproblem 1 is solved with the minimum number of processing-steps $\Phi$, which is calculated by adding up the minimum number of processing-steps of all vertices.

$$
\Phi=\sum_{v \in V} \phi_{v}
$$

The minimum number of processing-steps $\Phi$ ensures a feasible solution is always found. The solution given by subproblem 1 at this step provides an incumbent solution, and hence the initial upper bound (UB) of the search tree.

The search is conducted in a best first search strategy using a priority queue whose elements are always sorted ascendingly by the travelling times of their sequences. The queue is initialized by all TSP sequences (i.e. all Hamiltonian tours of the input graph) whose travelling times are smaller than UB.

At each iteration, the sequence at the head of the queue is considered. If the pruning condition in lemma 1 is satisfied, the node is pruned. Otherwise, the branching is conducted by generating and inserting all children of the sequence 
considered into the priority queue so that the ascending order of travelling times is still respected. In this manner, by the time the search terminates, the travelling time of the last sequence processed is the valid lower bound for the rest of the queue.

Everytime the number of processing-steps of the considered sequence is larger than the processing-steps of the best solution, the MIP model in subproblem 1 is solved with the new number of processing-steps to obtain a new ITSP solution. If the new solution is better than the best solution, the best solution and UB are then updated accordingly. The search terminates when the gap between the UB and LB is closed or all nodes have been searched.

Since during the branching, different paths can lead to the same sequence, explored sequences are kept in a visited archive which is updated incrementally during the search. The algorithm is described in algorithm 1.

In line 1 , the MIP model in subproblem 1 is solved with the minimum number of processing-steps $\Phi$. The resulting solution is the first incumbent solution which provides the initial upper bound $U B$. In line 3 , lower bound $L B$ is obtained by solving the TSP on the original instance using Concorde (Applegate et al., 2006). If the gap is not closed after the initial phase, the priority queue $Q$ is initialized with TSP sequences whose travelling times are smaller than $U B$ (line 7 ). The visited archive is initialized in line 8 to keep track of the visited sequences. The search is repeated until $Q$ is emptied or the gap is closed. At each search step, the sequence at the head of the queue is selected and removed from the queue (line 10). $L B$ is then updated accordingly (line 11 to 13 ). The LP model in subproblem 2 is solved to obtain the corresponding ITSP solution (line 14). If the number of processing-steps of the obtained solution is larger than the number of processing-steps of the best solution, the MIP model in subproblem 1 is solved with the new number of processing-steps (line 16). The best solution and UB are then updated accordingly (line 17-19). If lemma 1 is not satisfied, the current sequence is branched, i.e. children sequences are generated and inserted into the queue (line 21 to 29). Otherwise, the branching stops and the current sequence is pruned.

\section{Variable Neighborhood Search}

Variable Neighborhood Search (VNS) (Mladenović and Hansen, 1997) is a metaheuristic framework based on the systematic change of neighborhoods during the search in order to escape from the valleys containing local optima. The basic VNS uses a set of neighborhood structures denoted by $N_{k}, k=\left\{1, . ., k_{\max }\right\}$. At each iteration, a random solution from the $k^{t h}$ neighborhood is generated. A local search procedure, which takes this random solution as the starting point, is then called to obtain a local optimum. The process repeats until some stopping criteria are met. The basic VNS framework is described in algorithm 2 . A recent survey on the VNS can be found in (Hansen et al., 2010). The VNS has been successfully applied to many variants of the TSP (Sousa et al., 2015; Meng et al., 2018) and Vehicle Routing Problems (VRPs) (Wen et al., 2011; Pop et al., 2014).

In this paper, we use the general VNS (GVNS) (Hansen and Mladenović, 2002; Hansen et al., 2008), a variant of the VNS where the Variable Neighborhood Descent (VND) is used as the local search routine. The usual strategy 


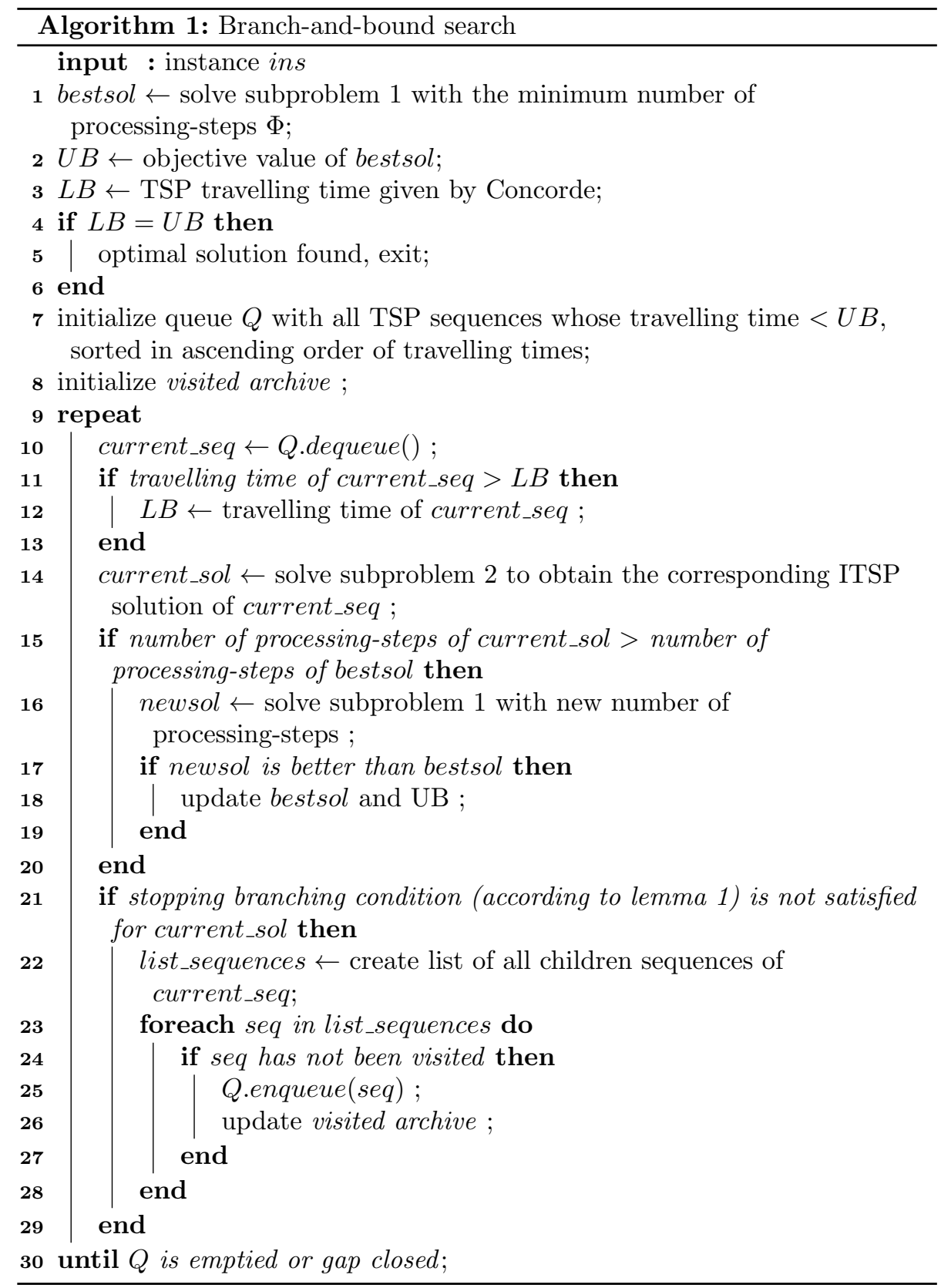




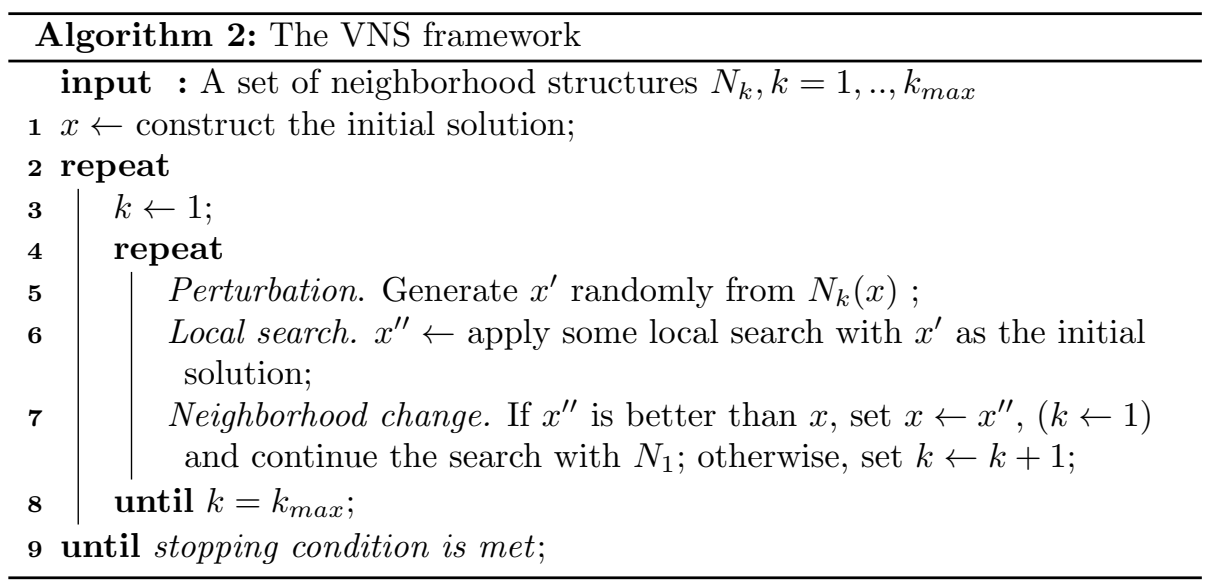

for exploration of several neighborhoods within deterministic local search is sequential (Seq-VND) (Hansen et al., 2008), where a set of neighborhoods are used in the search. Local searches are performed in a pre-defined order of this set. Everytime a better solution is found, the search returns to the first neighborhood in the list. The search stops when no improvement is found by any neighbourhoods.

In the following sections, we define the necessary components to apply the GVNS for the ITSP: initial solution constructor, local search procedure and perturbation procedure.

\subsection{Solution encoding}

Before describing the VNS components, we hereby present the solution representation. A solution is encoded as a list of processing-steps. Each processing-step $j$ consists of information of the corresponding vertex's id $(v)$, processing time $\left(p_{j}\right)$ and waiting time $\left(w_{j}\right)$ and the time that the processing actually starts $\left(t_{j}\right)$, i.e. the arrival time plus waiting time. To speed up the calculation, for each vertex, a list of corresponding processing-steps is also recorded. The objective value of a solution is the total processing time, i.e., the sum of the starting time and the processing time of the last processing-step. An illustration of a solution encoding can be found in figure 3 .

\subsection{Initial solution constructor}

Two constructive heuristics are proposed, namely NearestVertex and LongestVertex.

NearestVertex This heuristic starts from a random vertex, progressively processes the current vertex maximally (e.g. until the maximum temperature is reached) and walks to a random vertex in a list of nearest vertices where processing can start before the cooling has stopped on the current one. If no such vertex exists, the processing is continued at the same vertex after a proper waiting time. The algorithm is described in algorithm 3. 


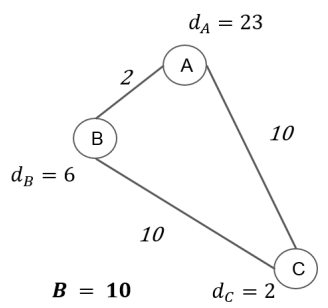

\begin{tabular}{|c|c|c|c|c|c|}
\hline $\begin{array}{c}\text { Processing } \\
\text {-step } j\end{array}$ & 0 & 1 & 2 & 3 & 4 \\
\hline Vertex $v$ & $\mathrm{~A}$ & $\mathrm{~B}$ & $\mathrm{~A}$ & $\mathrm{~A}$ & $\mathrm{C}$ \\
\hline$p_{j}$ & 10 & 6 & 10 & 3 & 2 \\
\hline$w_{j}$ & 0 & 0 & 0 & 3 & 0 \\
\hline$q_{j}$ & 0 & 0 & 0 & 10 & 0 \\
\hline$t_{j}$ & 0 & 12 & 20 & 33 & 46 \\
\hline \multicolumn{5}{|c|}{$S=p_{4}+t_{4}=48$} \\
\hline
\end{tabular}

Figure 3: Solution encoding for an instance with 3 vertices. The solution includes 5 processing-steps where vertex $A$ is visited three times.

LongestVertex The heuristic picks a random vertex from the list of $\alpha$ vertices with the longest remaining processing time. The processing of this vertex is combined with the complete processing of other vertices, in order to facilitate the cooldown of the current vertex, until it has been fully processed. The heuristic is described in algorithm 4 .

\subsection{Local search}

The local search procedure we choose is the Sequential VND (Hansen et al., 2008), where a set of neighborhoods is used. The Sequential VND iteratively selects a move and calls the corresponding first improvement hill climbing local search to obtain a local optimum which is then used as the starting point for the next iteration. The search terminates when no improvement is found by any neighborhood moves.

Thanks to the similarity in TSP and ITSP solution structures, neighborhood classes from the TSP are applicable for the ITSP. The following moves are used in our algorithm: 2-opt (Croes, 1958), 3-opt (Bock, 1958) (Lin, 1965), or-opt and remove-and-reinsert (Nilsson, 2003).

Due to the temperature constraints, evaluating the benefit of a move is costly. Therefore, we propose to estimate an ITSP move before actually proceeding with the move. We illustrate this estimation using the 2-opt move as follows. Consider the TSP 2-opt move where edges $(j, j+1)$ and $(k-1, k)$ are replaced by $(j, k-1)$ and $(j+1, k)$ and the segment $(j+1, k-1)$ is reversed (see figure 4 ), the move yields a benefit if $c_{j, j+1}+c_{k-1, k}-\left(c_{j, k-1}+c_{j+1, k}\right)>0$ (in symmetric instances). Therefore, checking if a move is beneficial in the TSP is $O(1)$.

In the ITSP, due to the temperature constraints, arrival temperatures, starting times and waiting times of all processing-steps starting from $k-1$ have to be recalculated. The new objective value is estimated by only considering processing-steps within an estimated radius $r$ around $j$ and $k$. The rational for this estimation is that if there is a change in visiting times due to the temperature constraints, it is most likely caused by processing-steps in a small radius around the processing-steps involved in the move, since processing-steps of the same vertices tend to be grouped together in a small segment. The estimation and the reasoning for it is illustrated in figure 4 where $j=3, k=10$ and the move reverses the segment from 4 to 9 . In the new solution, time information 

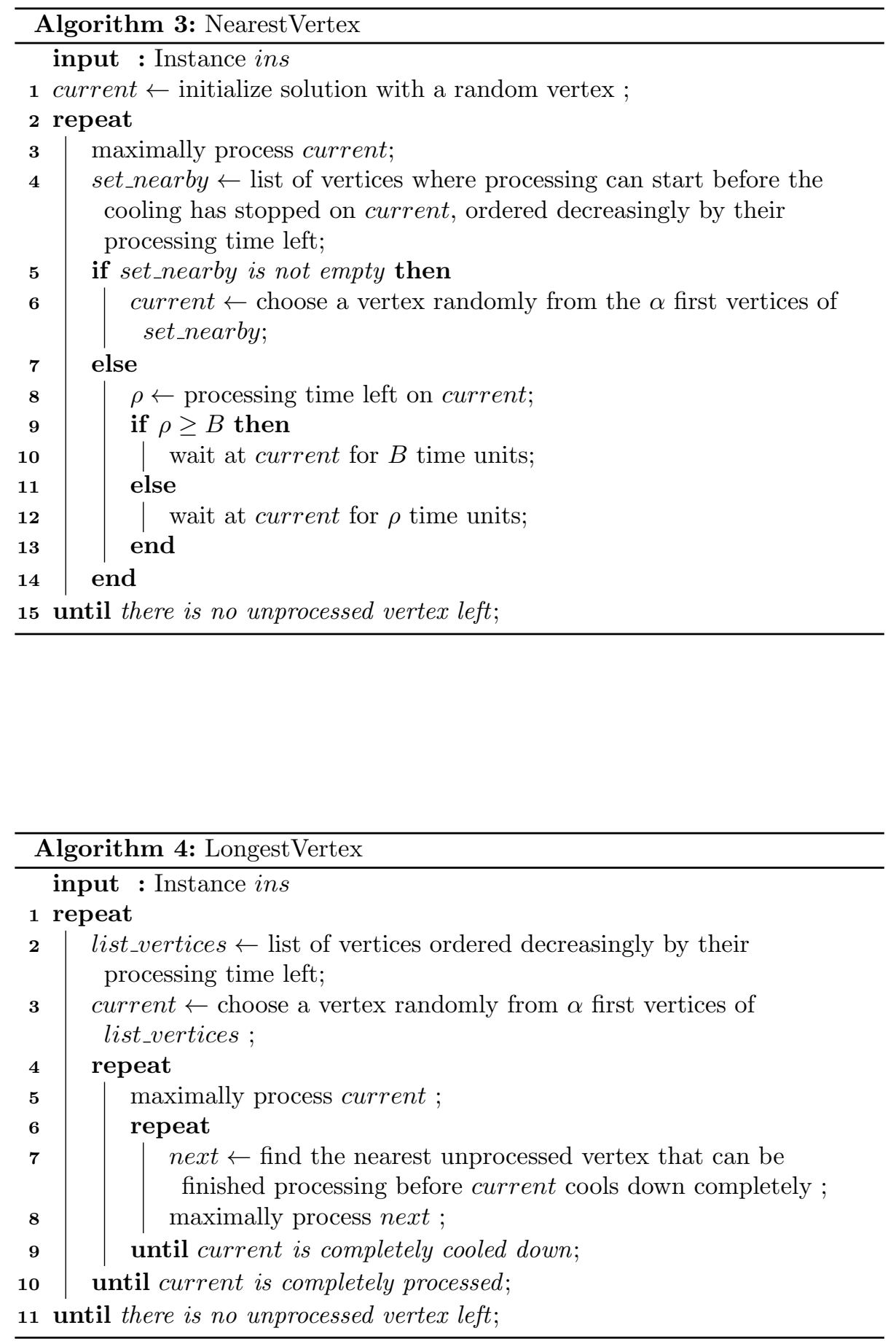
(starting time and waiting time) of processing-steps starting from 9 must be recalculated. To quickly estimate the move, only time information of the segment $(k-1, k-r)$ (processing-steps 9 and 8 in the example) are recalculated. The time difference between the old solution and the new solution of $k-r$ is forwarded to processing-step $k$ (processing-step 10 in the example). Time information of processing-steps in segment $(k, k+r)$ (processing-steps 10, 11, 12) is then calculated, taking into account the forwarded time difference. The difference between the starting time of processing-step $k+r$ in the old solution and $k+r$ in the new solution (processing-step 12 in the example) will give an estimated delta objective to evaluate the move.

To calculate the new time information of processing-step 9 in the new sequence, we need to look back to the processing-steps before it, i.e. processingsteps starting from 3 backwards to the beginning of the sequence, to check if temperature constraints are violated. In the estimation, we look back only $r=2$ processing-steps before 3. As one can observe, the larger $r$ is, the more precise the estimation becomes.

If the estimated objective value is smaller than the original one, i.e. the move might be beneficial, we proceed with the move and recalculate the whole solution to get the exact objective value. A similar scheme is applied for other moves.

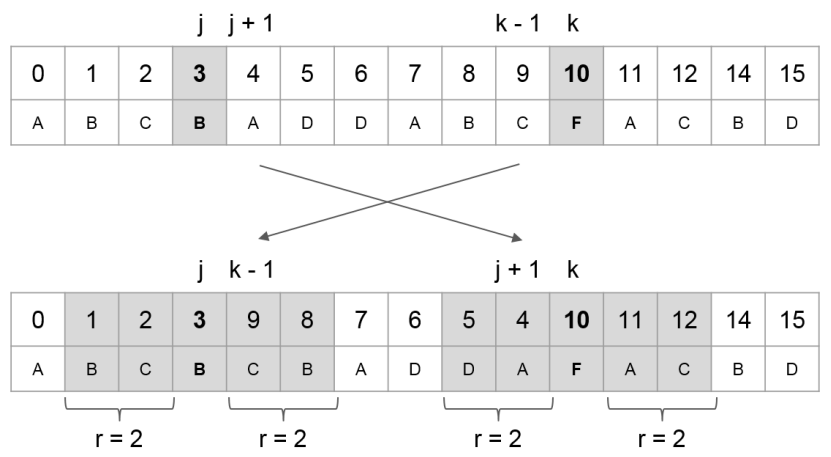

Figure 4: Move evaluation example.

For each neighborhood move, a first improvement hill climbing search is developed. When a local optimum is reached, the processing time of each processing-step is probably not optimal for the given sequence anymore. Therefore, a post processing is applied to adjust the processing time of each processingstep. This is done by solving the LP model in section 3.1 for the corresponding sequence.

\subsection{Perturbation}

Perturbation plays an important role in guiding the search to exploit new regions of the search space. Starting at the local optimum given by the local search procedure, perturbation makes a large move to jump to a distant neighbor in order to escape from the corresponding valley. We propose two perturbation moves for the framework: ruin-and-recreate and modified hyperedges exchange. 
Ruin-and-Recreate (RR) A $R R$ move (Schrimpf et al., 2000) includes two phases: the ruin phase removes some vertices from the tour while the recreating phase inserts those vertices into the tour. The $R R$ for the ITSP is conducted as follows: In the ruin phase, a seed vertex chosen randomly is removed from the solution. Then a number of its closest vertices are also removed. Afterward, the recreate phase reinserts those vertices back into the tour using the best insertion strategy. The parameter of the $R R$ move is the percentage of vertices being removed from the route.

Guided hyperedges exchange A hyperedge $H=\left(a_{0}, h\right)$ includes $h$ consecutive edges and starts from vertex $a_{0}$. In hyperedges exchange, two hyperedges $H_{1}=\left(a_{0}, h\right)$ and $H_{2}=\left(b_{0}, h\right)$ are removed from the tour leaving their vertices isolated. Those vertices are then reconnected to the tour randomly. The hyperedges exchange move is illustrated in figure 5 .

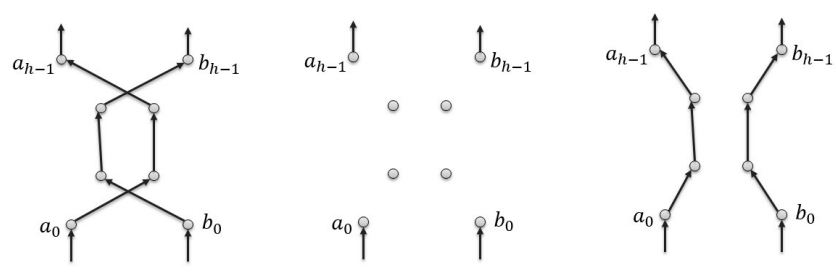

Figure 5: Hyperedges exchange.

In order to guide the algorithm to a promising region of the search space, we adopt the guided perturbation of (Burke et al., 2001) in the ITSP by utilizing information of the current tour. The quality of a hyperedge $H=\left(a_{0}, a_{1}, . ., a_{h-1}\right)$ is evaluated by:

$$
\sum_{j \in H}\left(c_{j, j+1}-\min _{v \in V \mid v \neq v_{j}} c_{v_{j}, v}\right)+\mu \sum_{j \in H} w_{j}
$$

with $w_{j}$ the waiting time at processing-step $j$ and $\mu$ the normalizing factor. The first term of the formula measures the difference between the total distance from each visit to its successor in the hyperedge and to its closest neighbor. A large difference indicates that there might be a chance to obtain a shorter path by destroying and re-routing that path. The second aggregates the waiting times along the hyperedge. The reasoning behind this term is that if we have to wait for a long time at a vertex, then it might be better to visit other vertices instead of waiting there. The role of $\mu$ is to distribute the influence of the two terms equally. The value of $\mu$ is set as the ratio between the first term and the second term of the first hyperedge whose aggregate waiting time is positive (denoted by $\left.H^{*}\right)$.

$$
\mu=\frac{\sum_{j \in H^{*}}\left(c_{j, j+1}-\min _{v \in V \mid v \neq v_{j}} c_{v_{j}, v}\right)}{\sum_{j \in H^{*}} w_{j}}
$$

In total, a high value of the function implies a poor quality hyperedge.

In guided hyperedges exchange, hyperedges are sorted increasingly by their quality values. At each perturbation step, a hyperedge is chosen randomly from the first $\theta$ percent of hyperedges in the list while the second hyperedge is chosen completely randomly. 


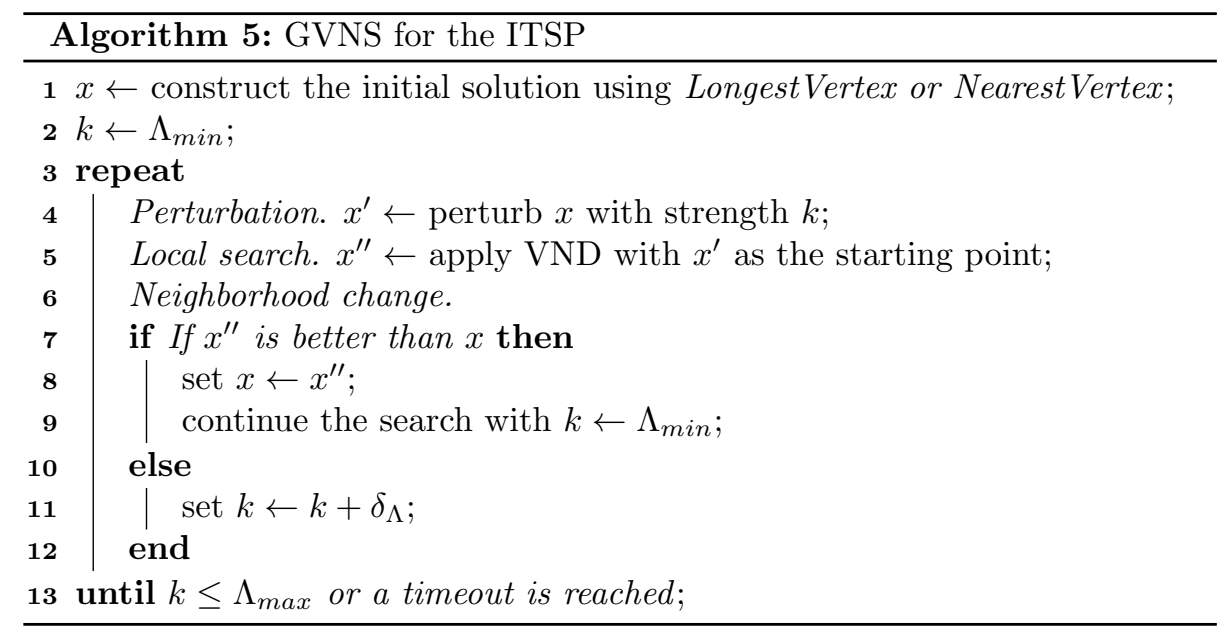

\subsection{The GVNS for the ITSP}

Given the components described earlier, the GVNS for the ITSP is conducted as follows. The neighborhood change is given by different strength of perturbation. Perturbation strength $k$ is initialized with its minimum value $\Lambda_{\min }$. At each iteration, a random solution is generated by perturbing the current solution with strength $k$. This solution serves as the starting point for the Seq-VND procedure. If a better solution is found, the incumbent solution is updated and $k$ is reset to $\Lambda_{\min }$. Otherwise, the solution is perturbed again with the same strength. After $n$ perturbations without improvement, where $n$ is the number of vertices of the instance, $k$ is increased with a step of $\delta_{\Lambda}$. The search terminates when $k$ exceeds its maximum allowed value $\Lambda_{\max }$ or a timeout is reached. Our GVNS algorithm for the ITSP is described in algorithm 5 .

Given two construction heuristics and two perturbation types, four different VNS metaheuristics are created. The two construction heuristics are named Nearest (NearestVertex) and Longest (LongestVertex) while the two perturbation types are named $R R$ (Ruin-and-Recreate) and Exchange (guided hyperedges exchange). Their combinations result in four different VNS metaheuristics: NearestRR, NearestExchange, LongestRR and LongestExchange respectively.

\section{Experiment}

\subsection{Test instances}

Since the problem is new in literature, we introduce a problem library consisting of two sets: set $R$ includes instances generated randomly and set $T$ includes instances generated from instances of the TSPLIB (Reinelt, 1991). All instances and best results are made publicly available at https://set.kuleuven.be/codes/ itsp_page.

There are two important features influencing the difficulty of an instance: (1) bound ratio which is the ratio between the maximum temperature $(B)$ and the average travelling time between vertices $(\bar{C})$. (2) instance density which 
Table 1: Density setting configuration

\begin{tabular}{l|lllllllllll} 
& D0 & D1 & D2 & D3 & D4 & D5 & D6 & D7 & D8 & D9 & D10 \\
\hline single-step (\%) & 100 & 90 & 80 & 70 & 60 & 50 & 40 & 30 & 20 & 10 & 10 \\
2-steps (\%) & 0 & 10 & 20 & 30 & 40 & 40 & 50 & 60 & 70 & 70 & 70 \\
3-steps (\%) & 0 & 0 & 0 & 0 & 0 & 10 & 10 & 10 & 10 & 20 & 10 \\
4-steps (\%) & 0 & 0 & 0 & 0 & 0 & 0 & 0 & 0 & 0 & 0 & 10
\end{tabular}

determines the number of vertices of each type. In our test instances, there are 4 types of vertices: 1, 2, 3 and 4-steps. A $\gamma$-step vertex needs at least $\gamma$ processingsteps to finish, i.e. its processing time $p$ lies in the range $(\gamma-1) B<p \leq \gamma B$. The number of vertices of each type in an instance is determined by its density setting. There are in total 11 density settings from $D 0$ to $D 10$. Lower density setting results in sparser instance. The detailed percentages of each type of vertex in each density setting are given in table 1 .

In set $R$, vertex coordinates are generated randomly in a square box. In set $T$, vertex coordinates are taken from the corresponding TSPLIB instance. Then, given a bound ratio and a density setting, processing times of vertices are generated accordingly. Instances are named after their size and settings. For example, an instance with size of 20 vertices, density setting $D 0$ and bound ratio 10 is named 20_D0_10. In case several instances have the same setting, an index is appended at the end of their names, e.g. 20_D0_10_1 and 20_D0_10_2. Names of instances in set $T$ includes their original name. E.g. instance 77_D9_10_pr76 is generated from instance $p r \% 6$, consists of 77 vertices (including the depot), has density setting $D 9$ and bound ratio 10 . Similarly to set $R$, an index is used to distinguish instances generated from the same TSPLIB instance and sharing the same setting. Both set $R$ and $T$ include many subsets for different experiments which will be presented in more detail in the following sessions.

The experiment consists of two parts. In the first part, we evaluate the performance of the branch-and-bound (BNB) approach and analyse problem difficulty regarding their settings using small instances in set $R$. In the second part, the VNS approach is evaluated using instances from both sets $R$ and $T$. All algorithms are implemented in Java. The MIP solver used is Gurobi 6.0.5 (Gurobi Optimization, 2015). Tests for the BNB algorithm are carried out on a Core i7, 2.9 GHz Intel machine with $7.7 \mathrm{~GB}$ of RAM. Tests for the VNS are carried out on a Linux-based PC cluster, with Intel Xeon E5-2680v3 $2.5 \mathrm{GHz}$ CPUs and a budget of $9 \mathrm{~GB}$ of RAM for each core.

\subsection{Evaluation of the branch-and-bound approach}

Three different experiments are carried out for different purposes. Three subsets of instances (R1, R2 and R3) with different sizes and settings are generated consequently.

In the first experiment on subset R1, the BNB performance with different instance sizes is evaluated. Instance size ranges from 6 to 12 vertices. For each size, 22 instances are generated corresponding to the combination of 11 density settings and 2 bound ratio settings (1 and 100).

In the second experiment on subset R2, we aim to analyse problem difficulty regarding bound ratio setting. Test instances have bound ratio ranging from 0.1 
Table 2: Instance configuration of set $R$

\begin{tabular}{lllll}
\hline Subset & No. instances & Instance size & Bound ratio & Density \\
\hline R1 & 154 & $6,7,8,9,10,11,12$ & 1,100 & D0 to D10 \\
R2 & 200 & 10 & $0.1,0.2,0.4, \ldots, 51.2(10$ settings $)$ & D2 \\
R3 & 220 & 10 & 10 & D0 to D10 \\
\hline
\end{tabular}

to 51.2 , the next ratio is double the current ratio $([0.1,0.2,0.4, \ldots 25.6,51.2])$. Instance size and density setting are fixed as 10 vertices and D2 respectively for all instances. For each bound ratio, 20 instances are generated.

In the third experiment on subset R3, we analyse problem difficulty regarding density setting. There are 11 density settings as listed in table 1 . Instance size is fixed as 10 vertices and bound ratio is 10 for all instances. For each density setting, 20 instances are generated. The summary of three subsets and their configurations can be found in table 2 .

A time limit of 1200 seconds is imposed on the MIP solver to solve subproblems and 3600 seconds on the BNB algorithm.

The results of this experiment are analysed in figure 6 and table 3 . Figure 6 shows the box plots of the running times of the algorithm on the three subsets while table 3 reports the average values of the running times, initializing times and the size of the queue for each subset, categorized by the corresponding targeted feature (instance size in R1, bound ratio in R2 and density setting in R3). In most of the test instances, optimal solutions are found, except some large instances in set $R 1$. In the experiment, if the running time of an instance exceeds the time limit (3600 seconds), its optimal solution is not found.

Figure 6a shows the results for experiment 1 on subset $R 1$ of instances with different sizes. We can see easily from the figure that problem difficulty depends highly on instance size (which is predictable). The corresponding initializing times and queue sizes given in table 3 show that the larger an instance is, the longer it takes to initialize the queue. This is caused by the exponential increase in the number of TSP sequences when the instance size increases. This suggests that the BNB approach is not scalable for big instances.

Figure $6 \mathrm{~b}$ shows the results for experiment 2 on subset $R 2$ including instances with the same size and density setting but different bound ratio. As shown in the figure, problem difficulty increases when bound ratio increases. This can be explained as follows. A large bound ratio means a large difference between the maximum temperature $B$ and the average travelling time $\bar{C}$, which leads to more waiting times in the solution. Since $L B$ is the total travelling time of the solution and $U B$ is the sum of the total travelling time and the aggregate waiting time, a large aggregate waiting time results in a large gap between $L B$ and $U B$. A large gap makes an instance difficult for the BNB algorithm to solve. This also results in a bad cut-off value for the initializing process, which is reflected in the large initializing times and queue sizes in instances with large bound ratio in table 3 . This explains why instances with high bound ratio settings tend to be more difficult. This also explains the small differences when the bound ratio exceeds 2 . The larger the bound ratio is, the larger the aggregate waiting time in the solution is. However, when the bound ratio exceeds 2, the waiting times are mostly 0 and cannot go any lower. Therefore, given the same instance size, instances with bound ratio exceeds 2 have similar level of 
Table 3: Branch-and-bound results on the three subsets $R 1, R 2$ and $R 3$ respectively

\begin{tabular}{|c|c|c|c|c|c|c|}
\hline & \multicolumn{2}{|c|}{ Running time } & \multicolumn{2}{|c|}{ Initializing time } & \multicolumn{2}{|c|}{ Queue size } \\
\hline & Avg (seconds) & Std (\%) & Avg (seconds) & Std $(\%)$ & Avg (seconds) & Std $(\%)$ \\
\hline \multicolumn{7}{|c|}{ Instance size (R1) } \\
\hline 6 & 1.56 & 247.41 & 0.05 & 55.16 & 47.36 & 92.58 \\
\hline 7 & 2.11 & 180.45 & 0.17 & 76.86 & 136.91 & 115.02 \\
\hline 8 & 27.59 & 262.59 & 0.92 & 96.76 & 989.55 & 148.07 \\
\hline 9 & 146.54 & 201.74 & 5.07 & 114.18 & 4763.86 & 170.44 \\
\hline 10 & 453.66 & 127.03 & 47.01 & 142.99 & 58412 & 176.01 \\
\hline 11 & 1366.57 & 111.49 & 1096.72 & 142.4 & 196706.68 & 162.28 \\
\hline 12 & 1982.27 & 79.28 & 1845.38 & 89.4 & 44230.86 & 194.8 \\
\hline \multicolumn{7}{|c|}{ Bound ratio (R2) } \\
\hline 0.1 & 1.39 & 43.54 & 0.5 & 86.92 & 9.3 & 83.92 \\
\hline 0.2 & 2.38 & 68.44 & 0.99 & 106 & 41.95 & 94.21 \\
\hline 0.4 & 4.22 & 110.14 & 1.54 & 95.92 & 159.8 & 159.98 \\
\hline 0.8 & 3.66 & 93.09 & 1.11 & 86.14 & 125.5 & 181.24 \\
\hline 1.6 & 9.14 & 108.87 & 3.65 & 131.55 & 849.6 & 141.38 \\
\hline 3.2 & 17.97 & 64.99 & 7.33 & 102.9 & 1345.78 & 205.2 \\
\hline 6.4 & 10.3 & 105.59 & 4.89 & 127.75 & 830.26 & 185.93 \\
\hline 12.8 & 12.54 & 97.3 & 5.93 & 137.03 & 1901.35 & 172.96 \\
\hline 25.6 & 16.34 & 92.38 & 6.02 & 112.16 & 1958.75 & 163.95 \\
\hline 51.2 & 16.02 & 91.12 & 7.82 & 129.12 & 1929.05 & 171.5 \\
\hline \multicolumn{7}{|c|}{ Density setting (R3) } \\
\hline D0 & 0.85 & 57.41 & 0 & 0 & 0 & 0 \\
\hline D1 & 14.82 & 116.68 & 7.6 & 130.8 & 620.9 & 208.15 \\
\hline D2 & 29.2 & 64.76 & 11.44 & 80.16 & 1002.95 & 134.64 \\
\hline D3 & 81.44 & 98.12 & 42.63 & 146.54 & 10902.8 & 172.91 \\
\hline D4 & 135.93 & 81.11 & 45.14 & 107.24 & 12652.8 & 167.87 \\
\hline D5 & 778.6 & 58.78 & 133.93 & 70.58 & 57030.1 & 103.95 \\
\hline D6 & 1003.41 & 43.59 & 120.4 & 79.78 & 89603.8 & 95.05 \\
\hline D7 & 1142.34 & 32.23 & 96.66 & 62.74 & 128895.2 & 86.57 \\
\hline D8 & 1212.93 & 14.93 & 78.84 & 67.38 & 94191.5 & 100.81 \\
\hline D9 & 1350.65 & 3 & 152.95 & 22.23 & 232340.47 & 38.31 \\
\hline D10 & 1615.17 & 9.93 & 414.89 & 38.63 & 275203.7 & 37.05 \\
\hline
\end{tabular}

difficulty.

Figure 6c shows the results for experiment 3 on subset $R 3$ which aims to compare the BNB performance on different density settings. Predictably, the denser an instance is, the harder it is to solve. This can also be explained by the large aggregate waiting time in dense instances.

To give some more insight into how the BNB works, we analyse the detailed results of some selected instances from all 3 subsets in table 4 . Information reported includes: instance name, itsp size (e.g. minimum number of processingsteps), search status (FINISHED means optimum found, the queue is emptied within the time limit or the gap is closed), running time, queue initializing time, size of the search queue, lower bound, upper bound and finally solution gap. What stands out in the table is that in $D 0$ instances, the number of nodes in the search tree is 0 . This can be explained as follows. For Do instances where all vertices are 1-step vertices, a TSP solution makes a valid ITSP solution. Therefore, in those instances, the gaps are closed right at the initial phase when the lower bound and upper bound are obtained. That is why those instances are easy to solve, resulting in small solving times of less than 2 seconds. In contrast, in big instances, the queue sizes are large and the time taken to initialize the queue takes up all the time budget. This is the main reason why the BNB is not scalable to big instances. 


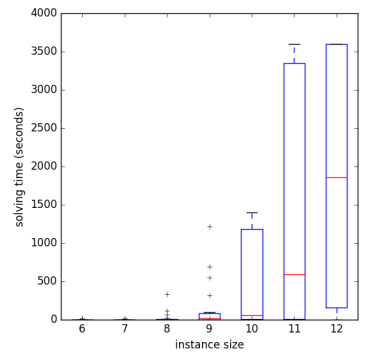

(a) Comparison on instance size,

subset R1.

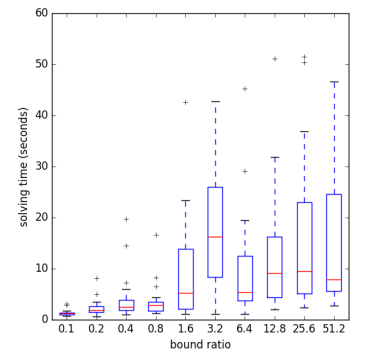

(b) Comparison on bound ratio,

subset R2.

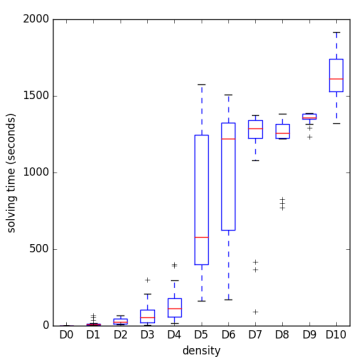

(c) Comparison on density setting, subset R3.

Figure 6: Branch-and-bound performance on different subsets.

Table 4: Detailed results on some selected instances in set $R$

\begin{tabular}{lllllllll}
\hline Instance & Itspsize & Status & Running time & $\begin{array}{l}\text { Queue initializing } \\
\text { time }\end{array}$ & Queue size & LB & UB & Gap \\
\hline 8_D9_100 & 16 & FINISHED & 466.67 & 2.56 & 4974 & 476 & 476 & $0.00 \%$ \\
8_D9_1 & 16 & FINISHED & 156.72 & 2.36 & 3084 & 406 & 406 & $0.00 \%$ \\
9_D0_100 & 10 & FINISHED & 0.15 & 0 & 0 & 334 & 334 & $0.00 \%$ \\
9_D0_1 & 10 & FINISHED & 0.14 & 0 & 0 & 285 & 285 & $0.00 \%$ \\
10_D1_1 & 12 & FINISHED & 2.95 & 1.36 & 128 & 412 & 412 & $0.00 \%$ \\
10_D2_100 & 13 & FINISHED & 10.23 & 4.44 & 564 & 387 & 387 & $0.00 \%$ \\
10_D2_51.2_10 & 13 & FINISHED & 39.2 & 18.7 & 5328 & 488 & 488 & $0.00 \%$ \\
10_D2_51.2_11 & 13 & FINISHED & 5.72 & 1.94 & 318 & 361 & 361 & $0.00 \%$ \\
10_D6_10_5 & 10 & FINISHED & 172.67 & 71.22 & 57194 & 502 & 502 & $0.00 \%$ \\
10_D6_10_6 & 10 & FINISHED & 663.03 & 119.96 & 121862 & 498 & 498 & $0.00 \%$ \\
11_D3_100 & 15 & FINISHED & 31.5 & 5.05 & 452 & 356 & 356 & $0.00 \%$ \\
11_D3_1 & 15 & FINISHED & 4.17 & 0.99 & 191 & 315 & 315 & $0.00 \%$ \\
12_D0_1 & 13 & FINISHED & 1.47 & 0 & 0 & 422 & 422 & $0.00 \%$ \\
12_D4_100 & 17 & FINISHED & 2605.94 & 711.32 & 50962 & 486 & 486 & $0.00 \%$ \\
12_D4_1 & 17 & FINISHED & 443.75 & 129.63 & 2128 & 384 & 384 & $0.00 \%$ \\
12_D5_100 & 19 & TIMEOUT & 3600 & 3600 & 15611 & 348 & 598 & $41.81 \%$ \\
12_D5_1 & 19 & TIMEOUT & 3600 & 1255.68 & 220195 & 298 & 416 & $28.37 \%$ \\
12_D6_100 & 21 & TIMEOUT & 3600 & 3600 & 2734 & 395 & 633 & $37.60 \%$ \\
12_D10_100 & 26 & TIMEOUT & 3600 & 3600 & 2475 & 376 & 845 & $55.50 \%$ \\
\hline
\end{tabular}


Table 5: Parameters tuning

\begin{tabular}{llll}
\hline Parameter & Description & Test values & Best setting \\
\hline$\alpha$ & Construction heuristics' list length & $10,50(\%)$ & 10 \\
$r$ & Estimated radius for local search & 3,10 (processing-steps) & 3 \\
$\theta$ & Hyperedges list size proportion & $25,75(\%)$ & 75 \\
$\left(\Lambda_{\min }, \Lambda_{\max }\right)$ & Perturbation min strength and max strength & $(10,60),(30,90)(\%)$ & $(30,90)$ \\
$\delta_{\Lambda}$ & Perturbation strength step & $5,10(\%)$ & 10 \\
\hline
\end{tabular}

Table 6: The VNS performance on set $\mathrm{R}$

\begin{tabular}{c|cccc} 
& NearestRR & NearestExchange & LongestRR & LongestExchange \\
\hline R1 & $0.00 \%$ & $0.00 \%$ & $0.08 \%$ & $0.08 \%$ \\
R2 & $0.00 \%$ & $0.00 \%$ & $0.16 \%$ & $0.17 \%$ \\
R3 & $0.00 \%$ & $0.00 \%$ & $0.01 \%$ & $0.01 \%$
\end{tabular}

\subsection{Calibration of the metaheuristics}

In order to tune the metaheuristic parameters, a tuning set is created by selecting instances from all subsets of set $R$ and $T$ in such a way that instances of all sizes and configurations are used. The size of the tuning set is 50 instances. For each parameter, 2 values are selected in a way that they cover most of its value ranges. The combination of 5 sets of parameters, with 2 values for each, results in 32 experiments. Note that $\theta$, the hyperedge list size proportion, is used in NearestExchange and LongestExchange only. For each instance, each experiment, the four metaheuristics (NearestRR, NearestExchange, LongestRR and LongestExchange) are applied for 10 runs with a time limit of 100 seconds for each. The best setting is selected from the combination which gives the smallest average deviation from best solutions. However, the one-way ANOVA test performed on the results shows that there is no significant difference between different parameter settings $(F=0.08, p=0.99)$. The parameters, their tested values and the best settings are listed in table 5 .

\subsection{Results and metaheuristics comparison}

To evaluate and compare the four metaheuristics proposed in this paper, three experiments are conducted. The results of all experiments in this section are the average of 5 runs.

In the first experiment, the four metaheuristics are tested on all three subsets of set $R$ which consists of small randomly generated instances. A time limit of 5 seconds is imposed on all of them. Table 6 shows the average deviation from the optimal solution for each metaheuristic and instance set. It can be seen from the table that all metaheuristics perform very well on those instances, especially NearestRR and NearestExchange with the average deviation of $0 \%$ in all instance sets. LongestRR and LongestExchange show small deviations especially in set $R 2$ but is still less than $0.2 \%$.

For the second experiment, a set of special instances $T 1$ are generated from the TSPLIB in a way that the optimal solutions are known. From a TSPLIB instance, a special instance is generated by setting the maximum temperature equal to 100 , and the processing time of each vertex equal to 1 . In such in- 
stances, each vertex is visited exactly once. Therefore its optimal solution is the optimal TSP solution (which is known) plus the number of vertices. T1 includes 80 instances corresponding with 80 TSPLIB instances with the size ranging from 14 to 3028 vertices. The time limit is set to 1800 seconds. The results for the second experiment are shown in figure 7 . The first plot shows the deviation of the metaheuristics' results from the optimal solutions while the running time is showed in the second plot. The results are good on NearestRR and NearestExchange with average optimality gaps of 4.09 and 4.21 respectively while LongestRR and LongestExchange, with average gaps 13.39 and 14.14 respectively, reveal inefficiency in large instances.
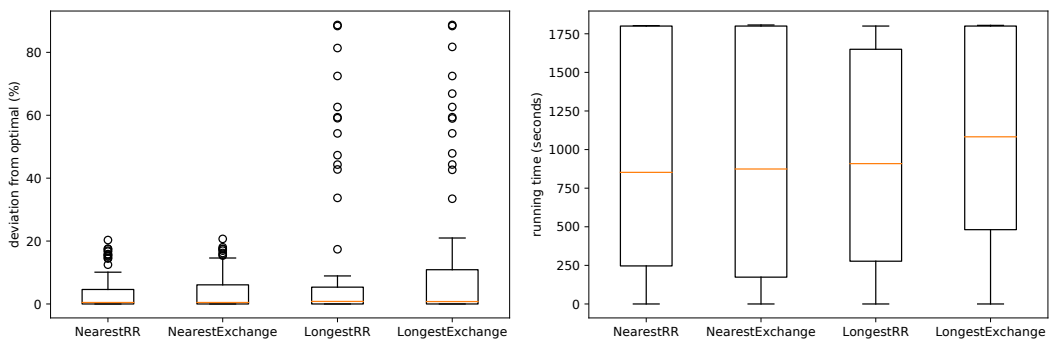

Figure 7: Results on special instances of set $\mathrm{T} 1$.

In the third experiment, the four metaheuristics are tested on big instances of set T2. 30 instances from the TSPLIB are randomly selected to generate instances in this set. For each instance, we generate 10 instances corresponding to the combination of density settings $D 0, D 2, D 4, D 7, D 9$ and bound ratio settings $0.1,10$. Therefore, set T2 consists of 300 instances. The sizes of instances in set T2 vary from 49 to 5916 vertices and the number of minimum processingsteps (which is called the ITSP-size) ranges from 49 to 12422 . Similarly to the second experiment, a time limit of 1800 seconds is imposed.

Since optimal solutions are unknown for those instances, we compare the results with the best known solutions obtained so far for each instance. In table 7 , the results are reported by groups of instances. Reported information includes the average deviation from the best know solutions and average running time. The first row of the table shows the results of each metaheuristic on the whole set. The next four rows show the detailed performances of each metaheuristic on each subgroup of set $T$ corresponding to 5 different density settings $(D 0$ to $D 9)$. The last two lines show results on 2 subsets corresponding to 2 bound ratio settings $(0.1$ and 10$)$. The results diverge more on instances with a small bound ratio.

A one-way ANOVA test on the results shows a statistically significant difference between the four algorithms $(F=43.52, p<0.001)$. It can be seen that NearestRR performs the best among the four metaheuristics. Paired-samples $t$-tests between NearestRR and the other three metaheuristics all give p-value less than 0.001 .

The metaheuristics using Nearest Vertex outperform the ones using LongestVertex in general, especially in a small bound ratio setting (0.1). This can be explained by the inclusion of the NearestVertex starting solution. This leads to a better performance, because it is more worthwhile to move to vertices that 
Table 7: Metaheuristics performance on set T2

\begin{tabular}{|c|c|c|c|c|c|c|c|c|}
\hline & \multicolumn{2}{|c|}{ NearestRR } & \multicolumn{2}{|c|}{ NearestExchange } & \multicolumn{2}{|c|}{ LongestRR } & \multicolumn{2}{|c|}{ LongestExchange } \\
\hline & Avg. dev (\%) & $\begin{array}{l}\text { Avg. Time } \\
\text { (seconds) }\end{array}$ & $\begin{array}{l}\text { Avg. dev } \\
(\%)\end{array}$ & $\begin{array}{l}\text { Avg. Time } \\
\text { (seconds) }\end{array}$ & $\begin{array}{l}\text { Avg. dev } \\
(\%)\end{array}$ & $\begin{array}{l}\text { Avg. Time } \\
\text { (seconds) }\end{array}$ & $\begin{array}{l}\text { Avg. dev } \\
(\%)\end{array}$ & $\begin{array}{l}\text { Avg. Time } \\
\text { (seconds) }\end{array}$ \\
\hline Overall & 0.35 & 1187.52 & 0.76 & 1270.66 & 7.31 & 1266.16 & 7.95 & 1229.58 \\
\hline \multicolumn{9}{|l|}{ Density } \\
\hline D0 & 0.48 & 949.49 & 1.24 & 1003.1 & 6.04 & 1040.01 & 7.01 & 985.58 \\
\hline D2 & 0.44 & 1197.03 & 0.91 & 1284.02 & 7.83 & 1299.87 & 8.47 & 1187.41 \\
\hline $\mathrm{D} 4$ & 0.28 & 1214.66 & 0.63 & 1288.11 & 6.96 & 1284.55 & 7.66 & 1269.08 \\
\hline D7 & 0.28 & 1271.36 & 0.51 & 1381.5 & 7.37 & 1384.1 & 7.87 & 1358.81 \\
\hline D9 & 0.24 & 1334.46 & 0.48 & 1429.08 & 8.55 & 1348.56 & 8.9 & 1373.51 \\
\hline \multicolumn{9}{|c|}{ Bound ratio } \\
\hline 0.1 & 0.68 & 1224.63 & 1.49 & 1249.34 & 14.51 & 1272.89 & 15.76 & 1245.03 \\
\hline 10 & 0.03 & 1151.44 & 0.06 & 1291.39 & 0.31 & 1259.62 & 0.35 & 1214.55 \\
\hline
\end{tabular}

are close by, rather than to move to vertices which have the longest remaining processing time. This holds especially for higher density settings, since in this case more visits to each vertex are required, resulting in higher travelling times for LongestVertex. Furthermore, Ruin-and-Recreate outperforms Hyperedges Exchange, because the former works with a closely connected subset of vertices, whereas the latter considers longer edges. Hence, it is shown that also for perturbation it is better to change the solution by considering vertices closest to those removed (ruin phase) rather than employing hyperedges. This results in a comparatively better performance of Nearest $R R$ and Longest $R R$ versus NearestExchange and LongestExchange respectively.

\section{Conclusion and future work}

In this paper we introduced a new variant of the TSP, namely the Intermittent Travelling Salesman Problem (ITSP), where a vertex may have to be visited more than once and there is a time lapse enforced between two consecutive visits due to the temperature constraint. An exact branch-and-bound approach is proposed to solve small instances. Instance difficulty regarding its properties is analysed based on the results from the exact approach. To tackle large instances, four VNS metaheuristics are built and compared with each other. An instance library consisting of two sets is built and made publicly available. The first set $R$ contains small, randomly generated instances while the second set $T$ contains large instances generated from instances of the TSPLIB. The results on small instances illustrate that all 4 metaheuristics result in very small deviation from optimal solutions, especially NearestRR and NearestExchange. On big instances, NearestRR performs the best on all instance sets while the performances of the other metaheuristics depend on instance properties.

The problem described in this paper is a simplification with respect to the real world setting in that we approximated the cooling schemes by linear functions and supposed them to be uniform over the workpiece. In future work, quadratic or exponential cooling and heating functions will be studied, as well as the influence of position dependent cooling and the interaction between nearby vertices. The simplified problem in its own right, even though it is shown to be accessible through usual combinatorial optimisation approaches, turns out to be particularly challenging. Interesting subjects to investigate in the future are stronger exact approaches, e.g. based on decomposition, for - approximately - solving larger instances. Heuristic approaches relying on alternative problem 
representations or approximate delta evaluation of the objective function also deserve further attention.

\section{References}

Applegate, D., Bixby, R., Chvatal, V., Cook, W., 2006. Concorde tsp solver.

Bock, F., 1958. An algorithm for solving "traveling salesman" and related network optimization problems, presented at the 14th national meeting of the operations research society of america, st. Louis, Missouri

Burke, E.K., Cowling, P.I., Keuthen, R., 2001. Effective local and guided variable neighbourhood search methods for the asymmetric travelling salesman problem. In Workshops on Applications of Evolutionary Computation, Springer, pp. 203-212.

Campbell, A., Clarke, L., Kleywegt, A., Savelsbergh, M., 1998. The inventory routing problem. In Fleet management and logistics. Springer, pp. 95-113.

Croes, G.A., 1958. A method for solving traveling-salesman problems. Operations research $6,6,791-812$.

Dumas, Y., Desrosiers, J., Gelinas, E., Solomon, M.M., 1995. An optimal algorithm for the traveling salesman problem with time windows. Operations research 43, 2, 367-371.

Gurobi Optimization, I., 2015. Gurobi optimizer reference manual; 2015. URL http://www. gurobi. com

Gutin, G., Punnen, A.P., 2006. The traveling salesman problem and its variations, Vol. 12. Springer Science \& Business Media.

Hansen, P., Mladenović, N., 2002. Developments of variable neighborhood search. In Essays and surveys in metaheuristics. Springer, pp. 415-439.

Hansen, P., Mladenović, N., Pérez, J.A.M., 2008. Variable neighbourhood search: methods and applications. 4OR 6, 4, 319-360.

Hansen, P., Mladenović, N., Pérez, J.A.M., 2010. Variable neighbourhood search: methods and applications. Annals of Operations Research 175, 1, $367-407$.

Johnson, D.S., McGeoch, L.A., 1997. The traveling salesman problem: A case study in local optimization. Local search in combinatorial optimization 1, $215-310$.

Laporte, G., 1992. The traveling salesman problem: An overview of exact and approximate algorithms. European Journal of Operational Research 59, 2, $231-247$.

Lin, S., 1965. Computer solutions of the traveling salesman problem. The Bell System Technical Journal 44, 10, 2245-2269. 
Meng, X., Li, J., Dai, X., Dou, J., 2018. Variable neighborhood search for a colored traveling salesman problem. IEEE Transactions on Intelligent Transportation Systems 19, 4, 1018-1026.

Mladenović, N., Hansen, P., 1997. Variable neighborhood search. Computers \& operations research 24, 11, 1097-1100.

Nilsson, C., 2003. Heuristics for the traveling salesman problem. Technical report, Tech. Report, Linköping University, Sweden.

Pop, P.C., Fuksz, L., Marc, A.H., 2014. A variable neighborhood search approach for solving the generalized vehicle routing problem. In International Conference on Hybrid Artificial Intelligence Systems, Springer, pp. 13-24.

Reinelt, G., 1991. Tspliba traveling salesman problem library. ORSA journal on computing 3, 4, 376-384.

Schrimpf, G., Schneider, J., Stamm-Wilbrandt, H., Dueck, G., 2000. Record breaking optimization results using the ruin and recreate principle. Journal of Computational Physics 159, 2, 139-171.

Sousa, M.M., Ochi, L.S., Coelho, I.M., Gonçalves, L.B., 2015. A variable neighborhood search heuristic for the traveling salesman problem with hotel selection. In Computing Conference (CLEI), 2015 Latin American, IEEE, pp. 1-12.

Wen, M., Krapper, E., Larsen, J., Stidsen, T.K., 2011. A multilevel variable neighborhood search heuristic for a practical vehicle routing and driver scheduling problem. Networks 58, 4, 311-322. 